\title{
Complication Rate of Endosonography (Endobronchial and Endoscopic Ultrasound): A Systematic Review
}

\author{
M.B. von Bartheld ${ }^{\mathrm{a}} \quad$ A. van Breda ${ }^{\mathrm{a}} \quad$ J.T. Annema ${ }^{\mathrm{a}} \mathrm{b}$ \\ ${ }^{a}$ Department of Pulmonology, Leiden University Medical Center, Leiden, and ${ }^{b}$ Department of Pulmonology, \\ Amsterdam Medical Center, Amsterdam, The Netherlands
}

\section{Key Words}

Adverse events - Complication rate · Cysts - Endobronchial ultrasonography · Endoscopic ultrasonography .

Fine needle aspiration - Mediastinum · Sarcoidosis .

Transbronchial needle aspiration · Ultrasound

\section{Abstract}

Background: Endosonography [endoscopic ultrasound (EUS)-guided fine needle aspiration and endobronchial ultrasound (EBUS)-guided transbronchial needle aspiration] is increasingly used for lung cancer staging and the assessment of sarcoidosis. Serious adverse events (SAE) have been reported in case reports, but the true incidence of complications is yet unknown. Objectives: To assess the rate of SAE related to endosonography and to investigate associated risk factors. Materials and Methods: PubMed, EMBASE and Cochrane libraries were searched for eligible references up to April 2012 and these included studies reporting on linear EUS or EBUS for the analysis of mediastinal/hilar nodal or central intrapulmonary lesions. Case series describing complications were excluded. Reported complications were classified into SAE or minor adverse events (AE). Results: 190 studies met the inclusion criteria. Information on follow-up was missing in half of the studies. In 16,181 patients, 23 SAE
(0.14\%) and $35 \mathrm{AE}(0.22 \%)$ were reported. No mortality was observed. SAE were more frequent in patients investigated with EUS $(0.30 \%)$ than in those investigated with EBUS (0.05\%). Infectious SAE were most prevalent $(0.07 \%)$ and predominantly occurred in patients with cystic lesions and sarcoidosis. In lung cancer patients, complications were rare. Discussion: Endosonography for intrathoracic nodal assessment seems safe for lung cancer patients and mortality has not been reported. For cystic lesions and sarcoidosis, there may be a small, but nonnegligible risk of infectious complications. The true incidence of SAE might be higher as accurate documentation of complications is missing in most studies.

(c) 2014 S. Karger AG, Basel

\section{Introduction}

In patients with lung cancer, endosonography is the method of choice for intrathoracic nodal staging according to current guidelines [1, 2]. Additionally, it seems the diagnostic method of choice for the assessment of sarcoidosis [3,4]. Several - mainly infectious (mediastinitis/abscess formation) - severe adverse events (SAE) have been reported in the literature raising

\section{KARGER}

E-Mail karger@karger.com

www.karger.com/res
(C) 2014 S. Karger AG, Basel

0025-7931/14/0874-0343\$39.50/0
J.T. Annema, MD, $\mathrm{PhD}$

Department of Pulmonology, Academic Medical Center, University of Amsterdam PO Box 22700

NL-1100 DE Amsterdam (The Netherlands)

E-Mail j.t.annema@amc.nl 
the debate on the safety of endosonography [5-10]. At present, data on the true incidence of endoscopic (EUS)/ endobronchial ultrasonography (EBUS) in routine use are scarce. With the rapid spread of novel endosonography techniques, careful monitoring for adverse events (AE) is important.

The aim of the current systematic review was to determine the incidence of SAE attributable to the use of endosonography (EUS and EBUS) for intrathoracic investigation and to identify subsets of patients who are at risk of developing SAE.

\section{Materials and Methods}

\section{Search Strategy}

We searched for articles and abstracts mentioning definitive information on complications of linear endosonography in the literature for both EUS and EBUS for real-time aspiration of mediastinal lymph nodes, and for mediastinal and centrally located intrapulmonary masses. An English language systematic literature search (from January 1989 to April 2012) was initiated using PubMed, Cochrane and EMBASE databases which included the following terms: 'endosonography', 'ebus', 'eus', 'endoscopic ultrasound', 'ultrasound-guided' combined with 'biopsy', 'fine-needle' combined with 'fine needle aspiration', 'fna', 'transbronchial needle aspiration', 'tbna' combined with 'mediastinum', 'lung', 'respirator', 'pulmonary' and 'sarcoidosis'.

All initial search results were firstly screened for the eligibility criteria (see below) by one investigator (A.v.B.) based on title and abstract. Subsequently, the full article of possibly eligible articles was further screened by the same criteria and if indeed eligible, data on the type of investigation, study design, sedation, needle size, complications and follow-up were documented by two authors (A.v.B. and M.B.v.B.). Articles with complications reported were further evaluated by two investigators (A.v.B. and M.B.v.B.). No effort was made to retrieve missing data from authors. Any discordance was solved by consensus agreement between all three investigators.

\section{Eligibility and Screening Criteria}

Considered for analysis were full-text, prospective and retrospective observational studies as well as (randomized) controlled trials and abstracts mentioning information on complications. All articles other than original datasets, such as case reports, case series with fewer than 5 patients, reviews, meta-analyses, duplicates, comments and editorials, were excluded from the analysis. In case no information was mentioned on the rate of complications, these articles were also excluded.

Studies assessing the role of EBUS and EUS for indications other than mediastinal or hilar lymph node aspirations or central lung masses (e.g. liver, adrenal gland, pancreatic pathology, esophageal cancer or duodenal lesions) were excluded. Also, studies of radial or guide-sheath EBUS assessing peripheral lung tumors were excluded. Furthermore, studies with patients $<18$ years, animals and cadavers were not eligible.

\section{Definitions of Outcomes}

Complications were defined as any AE occurring during and after the procedure and were classified into SAE and (minor) AE. An SAE consisted of any AE that was causally related to the procedure and was either lethal, could risk the patient's life, required hospital admission, inflicted permanent injury to the health of the patient and/or required intervention to prevent permanent impairment or damage [11]. AE required no hospital admission but did require either non- or minimally invasive interventions (e.g. blood oxygen saturation measurement, venous puncture or X-ray scanning) and they did not inflict permanent health damage, e.g. nausea, vomiting, chest pain, tachycardia, fever $<39^{\circ} \mathrm{C}$ and minor hemoptysis.

\section{Statistical Analyses}

The rates of both SAE and AE were calculated dividing the sum of the patients included by the number of reported complications. $\chi^{2}$ and Fisher's exact test were used for the analysis of categorical data. All analyses were performed with SPSS (version 17.0).

\section{Results}

\section{Literature Search}

The search yielded 2,748 references of which 1,776 were excluded after reviewing the title and abstract. After reading the full text, a further 782 papers were excluded as they were review articles $(\mathrm{n}=257)$, complications were not mentioned $(n=150)$, they were case reports or case series including fewer than 5 patients $(n=149)$ or they referred to analysis of peripheral pulmonary lesions by radial or guided-sheath EBUS $(n=56)$. Other reasons for exclusion are mentioned in figure 1 . A total of 190 articles remained for analysis.

\section{Study Characteristics}

The 190 articles were published between February 1995 and April 2012. Information on the primary indication for EUS/EBUS, the study design and the type of follow-up are listed in table 1. EUS-guided fine needle aspiration (FNA) was the method of investigation in 99 studies (52\%), EBUS-guided transbronchial needle aspiration (TBNA) was used in $81(43 \%)$, and in 10 articles (5\%), both techniques were used. The total number of patients investigated was 16,181 (EBUS: 9,119; EUS: 6,042, and both EBUS and EUS: 1,020 patients) with a mean of 85 patients per study (range 5-601). Midazolam was used as the sedation of choice in 87 studies (46\%), general anesthesia in $17(9 \%)$, pethidine in $21(11 \%)$, propofol in 11 (6\%) and other medication or a combination of anesthetics in 12 (6\%); in 42 studies (22\%), the type of sedation was unclear. The needle size used for tissue aspiration was 22 gauges in 124 studies (65\%), 21 gauges in 7 (4\%), 23 
Fig. 1. Flow chart for the selection of articles reporting $\mathrm{AE}$ of endosonography (EBUS and EUS) for mediastinal lesions.
2,748 references by initial search screened for relevancy (PubMed, EMBASE, Cochrane; 1989-2012)

1,776 articles excluded based on title and abstract

782 articles excluded after reading the full article based on no subject of interest:

257 review articles

149 case reports/series ( $<5$ patients)

150 complications not mentioned

56 peripheral pulmonary lesions

50 editorials/letters to the editor

40 no EUS/EBUS research

35 gastrointestinal indications

27 no original data

13 animal studies

5 cost-analysis studies

190 studies included gauges in 3 (2\%), 19 gauges in $3(2 \%)$ and 20 gauges in 1 (1\%), and a combination of needle sizes was applied in 27 studies (14\%). Trucut and forceps biopsies were used in $9(5 \%)$ and 3 studies (2\%), respectively. In 1 study (0.5\%), no FNA was performed and 24 studies (13\%) did not specify needle size.

\section{Reported Complications}

A total of 23 SAE (tables 2, 3) were reported in 36 studies including 16,181 patients, corresponding to an SAE rate of $0.14 \%$. Mortality was not reported. In those studies, 35 minor $\mathrm{AE}$ were observed $(0.22 \%)$, most of them ( 28 of $35 \mathrm{AE}$ ) were reported in 4 articles. In 151 studies (79\%), no AE were reported and in the remaining 7 (4\%) it was stated that there was at least no severe complication.

Eighteen SAE and $25 \mathrm{AE}$ were EUS complications, and EBUS-associated SAE and AE were seen in 5 and 10 patients, respectively. No AE were observed in studies combining EUS and EBUS. SAE rates for EUS and EBUS were $0.30 \%$ (18 in 6,042 patients) and $0.05 \%$ (5 in 9,119 patients), respectively. Of the $23 \mathrm{SAE}, 1$ (25\%) was extracted from a randomized trial ( $\mathrm{n}=4,246$ patients), $12(11 \%)$ were from nonrandomized prospective studies $(\mathrm{n}=109$, 9,066 patients) and 10 (13\%) were from retrospective studies ( $n=77,6,732$ patients). The respective SAE rates for randomized controlled trials, and prospective and retrospective designs were $0.41,0.13$ and $0.15 \%$. Of the 23 SAE in all 16,181 patients, $12(52 \%)$ were associated with infectious complications, 4 (17\%) with perforations of esophageal or pharyngeal structures, 3 (9\%) with respiratory insufficiency, 2 (9\%) with a pneumothorax and 2 (9\%) with a puncture-related hemorrhage. Severe complications occurred in 8 patients with lung cancer (35\%), in 5 with mediastinal cysts (22\%), in 3 with sarcoidosis (13\%), in 2 with tuberculosis (9\%), in 1 mesothelioma (4\%) and in 1 benign lymphadenopathy patient (4\%). In the remaining 3 patients (13\%), the indication for endosonography was unclear.

There were no reports of SAE-induced persistent impairment in the patients. Outcomes were reported as full recovery $(\mathrm{n}=11)$, unclear $(\mathrm{n}=1)$ and no information was given on the outcome in the remaining patients $(\mathrm{n}=11)$. 


\section{Severe Adverse Events}

\section{Infectious Complications}

Five patients had a diagnosis of mediastinitis and 3 had mediastinal abscess formation (tables 2, 3). Three of those were in patients with a final diagnosis of a mediastinal cyst. Two patients underwent Trucut biopsy of a mediastinal mass. The first was a 52-year-old man referred for evaluation of a bulky lower mediastinal mass. Six earlier passes with EUS-FNA yielded no definitive diagnosis so 3 Trucut biopsies were obtained. Two days later, the patient presented with complaints of dysphagia and chest pain. CT scanning showed an image compatible with mediastinitis. During thoracotomy, an 8 -cm cystic lesion with purulent drainage arising from the distal esophagus was excised. The patient recovered uneventfully and was discharged after 8 days. The other patient developed severe sepsis secondary to mediastinitis 4 days after EUSFNA. Thoracotomy was indicated and revealed an infected bronchogenic cystic abscess. The third patient had a lymph node abscess following EBUS-TBNA of a subcarinal cystic metastatic lymph node, but it resolved after antibiotic treatment. One patient with non-small cell lung cancer (NSCLC) and brain metastases developed mediastinitis after an iatrogenic esophageal perforation due to technical problems with the needle. He was treated with antibiotics, discharged 25 days after the procedure and died 6 days later due to progression of his malignancy. Another patient with a left upper lobe tumor and an enlarged, necrotic-appearing subcarinal lymph node underwent 4 FNA which yielded small cell carcinoma. Two days after EUS, he developed fever. Without an evident focus for the fever, he was given empiric antibiotic treatment, only to return 2 days later with persistent fever and elevated inflammatory parameters. CT scanning showed air bubbles in the subcarinal area for which intravenous antibiotics were started. After 1 week, the inflammatory parameters normalized and the patient was treated with concurrent chemoradiotherapy.

One patient with sarcoidosis underwent diagnostic FNA with a 19-gauge needle to obtain tissue proof of noncaseating granulomas. He complained of chest pain the day after the procedure and was diagnosed with mediastinitis based on blood examination as well as CT findings. His symptoms resolved during the 1-week treatment with antibiotics. Another sarcoidosis patient developed retrosternal pain and fever 2 days after EUSFNA of subcarinal and paratracheal lymph nodes but only reported 10 days after developing symptoms. CT images showed local abscesses in the biopsied area. Following necrotic nodal tissue removal during thoracoto-
Table 1. Characterization of the studies included $(n=190)$ regarding study design, indication for EUS/EBUS and follow-up

$\mathrm{n}$

$\%$

Study design

Randomized trial

Nonrandomized trial

Prospective

Retrospective

42

18698

$109 \quad 59$

$77 \quad 41$

Indication for EUS/EBUS

Diagnosis and/or staging of lung cancer $\quad 85 \quad 38$

Assessment of mediastinal lesions $\quad 55 \quad 29$

$\begin{array}{lrr}\text { Suspicion of sarcoidosis } & 12 & 6\end{array}$

Suspicion of tuberculosis $\quad 3 \quad 2$

$\begin{array}{llll}\text { Mediastinal staging of extrathoracal malignancies } & 7 & 4\end{array}$

$\begin{array}{lll}\text { Restaging of lung cancer } & 5 & 3\end{array}$

Diagnosis of mediastinal cysts $\quad 2 \quad 1$

Diagnosis of lymphoma $\quad 1 \quad 1$

$\begin{array}{lll}\text { Various indications } & 20 & 11\end{array}$

\section{Follow-up}

Follow-up mentioned $\quad 97 \quad 51$

Follow-up $\geq 1$ month $\quad 45 \quad 24$

Follow-up $\leq 1$ month $\quad 33 \quad 17$

Follow-up, but no specified length $\quad 16 \quad 8$

Follow-up of patient complaints $\quad 2 \quad 1$

No follow-up $\quad 1 \quad 1$

Follow-up not mentioned $\quad 93 \quad 49$

Table 2. Overview of SAE following EUS-FNA $(n=6,042)$ and EBUS-TBNA $(n=9,119)$ for mediastinal analysis $(n=23)$

\begin{tabular}{|c|c|c|}
\hline & $\begin{array}{l}\text { EUS } \\
(n=18)\end{array}$ & $\begin{array}{l}\text { EBUS } \\
(n=5)\end{array}$ \\
\hline Infectious complications $(\mathrm{n}=12)$ & 10 & 2 \\
\hline Mediastinitis & 5 & 0 \\
\hline Mediastinal abscess/abscess formation & 2 & 1 \\
\hline Sepsis & 1 & 1 \\
\hline Pleuropericarditis & 1 & 0 \\
\hline Aspiration pneumonia & 1 & 0 \\
\hline Perforations $(\mathrm{n}=4)$ & 4 & 0 \\
\hline Esophageal perforation/rupture & 3 & \\
\hline Sinus piriformis perforation & 1 & \\
\hline Pneumothorax $(\mathrm{n}=2)$ & 0 & 2 \\
\hline Hemorrhagic complications $(\mathrm{n}=2)$ & 2 & 0 \\
\hline Mediastinal hematoma & 1 & \\
\hline Periesophageal bleeding & 1 & \\
\hline Respiratory complications $(n=3)$ & 2 & 1 \\
\hline Hypoxemia due to airway edema & - & - \\
\hline Apnea under propofol & - & - \\
\hline Required reversal medication & 1 & 0 \\
\hline
\end{tabular}

Values represent numbers of patients. 
Table 3. SAE following EUS-FNA $(n=6,042)$ and EBUS-TBNA $(n=9,119)$
SAE [study]
Indication
Needle Interventions
Outcome
size, gauge

\section{EUS-FNA}

1 Mediastinitis (after esophageal NSCLC 22 perforation) [18]

22

mission (25 days) and treatment

patient died 6 days later with intravenous antibiotics due to progression of the malignancy

\begin{tabular}{|c|c|c|c|c|c|}
\hline 2 & Mediastinitis [19] & $\begin{array}{l}\text { small cell } \\
\text { lung cancer }\end{array}$ & 22 & $\begin{array}{l}\text { admission ( } 1 \text { week) and treatment } \\
\text { with intravenous antibiotics }\end{array}$ & recovery \\
\hline 3 & Mediastinal abscess with sepsis [20] & cyst & 22 & surgical treatment (not specified) & not reported \\
\hline 4 & Mediastinitis [21] & cyst & $\begin{array}{l}\text { Trucut } \\
\text { biopsy }\end{array}$ & admission ( 8 days) and thoracotomy & recovery \\
\hline 5 & Mediastinitis with sepsis [10] & cyst & $\begin{array}{l}\text { Trucut } \\
\text { biopsy }\end{array}$ & thoracotomy & not reported \\
\hline 6 & Pleuropericarditis [22] & cyst & 22 & $\begin{array}{l}\text { pleural drainage and admission } \\
\text { ( } 21 \text { days) }\end{array}$ & not reported \\
\hline 7 & Mediastinitis [23] & sarcoidosis & 19 & $\begin{array}{l}\text { admission and treatment with } \\
\text { intravenous antibiotics }\end{array}$ & recovery \\
\hline 8 & Mediastinal abscess formation [24] & sarcoidosis & 22 & thoracotomy & recovery \\
\hline 9 & Mediastinal abscess [25] & tuberculosis & $\begin{array}{l}\text { Trucut } \\
\text { biopsy }\end{array}$ & cervical mediastinal drainage & not reported \\
\hline 10 & Aspiration pneumonia [26] & NSCLC & unclear & $\begin{array}{l}\text { admission }(48 \mathrm{~h}) \text { and treatment } \\
\text { with intravenous antibiotics }\end{array}$ & not reported \\
\hline 11 & Esophageal perforation [27] & mesothelioma & 22 & $\begin{array}{l}\text { surgical correction of the } \\
\text { perforated esophagus and } \\
\text { several weeks of admission }\end{array}$ & not reported \\
\hline 12 & Esophageal perforation [18] & NSCLC & 22 & $\begin{array}{l}\text { admission ( } 6 \text { days) and treatment } \\
\text { with antibiotics }\end{array}$ & recovery \\
\hline 13 & Sinus piriformis perforation [28] & $\begin{array}{l}\text { goiter with } \\
\text { mediastinal } \\
\text { lymph- } \\
\text { adenopathy }\end{array}$ & 22 & $\begin{array}{l}\text { admission and treatment with } \\
\text { intravenous antibiotics }\end{array}$ & recovery \\
\hline 14 & Esophageal rupture [28] & NSCLC & 22 & $\begin{array}{l}\text { endoscopic clipping of the } \\
\text { esophagus }\end{array}$ & recovery \\
\hline 15 & Mediastinal bleeding [29] & NSCLC & unclear & fasting and bed rest & recovery \\
\hline 16 & Periesophageal bleeding [30] & $\begin{array}{l}\text { benign } \\
\text { lymph node }\end{array}$ & $\begin{array}{l}\text { Trucut } \\
\text { biopsy }\end{array}$ & $\begin{array}{l}\text { admission }(72 \mathrm{~h}) \text { and treatment } \\
\text { with intravenous narcotics }\end{array}$ & not reported \\
\hline 17 & $\begin{array}{l}\text { Respiratory failure (apnea) under } \\
\text { propofol [31] }\end{array}$ & unclear & unclear & $\begin{array}{l}\text { transient endotracheal intubation, } \\
\text { no admission }\end{array}$ & not reported \\
\hline 18 & Required reversal medication [32] & NSCLC & 22 & unclear & not reported \\
\hline
\end{tabular}

EBUS-TBNA

1 Mediastinal abscess formation [33] cys

22

2 Sepsis [34]

3 Pneumothorax [35]

4 Pneumothorax [36]

5 Airway edema/hypoxemia [37] cyst

tuberculosis 22

NSCLC

NSCLC

sarcoidosis admission and treatment with intravenous antibiotics

admission and treatment with recovery intravenous antibiotics

chest tube drainage recovery

chest tube drainage not reported

early termination of the procedure not reported 
my, the patient completely recovered. The last patient with a mediastinal abscess was a patient with a 4-month history of hoarse voice and weight loss undergoing EUSguided Trucut biopsy for tissue analysis of a paratracheal mass with echographic features suggestive of necrosis. Three days later, she presented with dysphagia and pyrexia, and CT revealed peritracheal and retroesophageal collections. Cervical drainage revealed pus. Initial EUSTrucut biopsy histology demonstrated histiocytic granulomas which were culture positive for Mycobacterium tuberculosis.

One procedure in a patient undergoing 22-gauge EUS-FNA for suspicion of a mediastinal cyst was complicated by a pleuropericarditis and atrial fibrillation. Treatment involved pleural drainage over a 21-day hospitalization.

Two patients developed sepsis. In 1 patient, puncture of a mediastinal cyst led to a septic complication, for which the patient was treated surgically. Another patient was scheduled for EBUS-TBNA to assess a right paratracheal lymph node. Four uncomplicated passes yielded necrotizing granulomas also positive on Ziehl-Neelsen staining. Under antituberculous therapy, the patient presented with sepsis 2 days after the procedure and blood cultures showed $\beta$-hemolytic group G streptococci. He improved with antibiotic treatment and was discharged after 6 days without further complications.

Finally, 1 patient was hospitalized after the procedure for aspiration pneumonia secondary to periprocedural aspiration of blood. The patient's condition improved with supportive care and intravenous antibiotics; the patient was discharged $48 \mathrm{~h}$ later.

Gastrointestinal Perforations and Pneumothoraces

Four perforations ( 3 esophageal and 1 sinus piriformis) related to EUS-FNA occurred on top of the one already mentioned above. One patient with mesothelioma was referred for surgical correction of an esophageal perforation and was discharged from hospital after several weeks. Another patient developed an esophageal perforation due to technical failure; he was treated with antibiotics and discharged 6 days after EUS-FNA in good clinical condition without developing an infectious complication. Furthermore, rupture of the esophageal wall occurred in 1 case because of improper use of the sheath of the needle. After the lesion was clipped, the patient's clinical course was uneventful. The remaining patient had swallowing problems and a large goiter. He was referred for analysis of mediastinal lymphadenopathy, and during EUS, rupture of the sinus piriformis was encountered due to difficulty in scope introduction. After administration of intravenous antibiotics, the patient recovered uneventfully.

Two patients undergoing EBUS-TBNA for diagnosis and staging of NSCLC developed a pneumothorax: 1 patient after FNA of a lung lesion and the other after aspiration of a mediastinal node. Both required chest tube drainage.

\section{Other Severe Complications}

Two patients had a complication involving mediastinal hematomas. One complained of pain after Trucut core biopsy of a benign subcarinal node. The bleeding was detected during EUS and the patient was observed for 3 days in the hospital and treated with intravenous narcotics for pain management. No significant drop in hemoglobin was seen. A CT scan showed a periesophageal hematoma but no intervention was necessary. The other patient had a mediastinal hematoma after EUS-FNA resolving after a conservative treatment of fasting and bed rest. One procedure was complicated by respiratory failure in a patient undergoing mediastinal EUS through the upper gastrointestinal tract under propofol sedation. $\mathrm{He}$ required transient endotracheal intubation but did not require hospitalization overnight. Another developed stridor and hypoxemia due to airway edema causing early termination of the procedure. A final patient required reversal medications, but no further information was given for this AE.

\section{Adverse Events}

A total of 35 minor complications were reported in the papers analyzed. During the examination in 4 EBUS patients, a small bleeding occurred at the puncture location, requiring instillation of $1 \mathrm{mg}$ noradrenaline to stop the bleeding in 3 patients; in 1 patient the procedure was terminated but the bleeding stopped without intervention. In 4 EUS patients, a small peri- or intranodal extravasate was seen on ultrasound images after nodal aspiration, but neither of them required intervention. In 1 patient, respiratory depression with apnea occurred. Abortion of the procedure resulted in full recovery. In another patient, nodal aspiration could not be performed due to intraprocedural oxygen desaturation. One patient developed stridor immediately after an EUS procedure, but no intervention was necessary. Two cases of atrial fibrillation (1 EUS and 1 EBUS procedure), 1 case of tachycardia ( $>150$ $\mathrm{bpm}$ ) and 1 case of hypotension were also described: all were self-limiting. In 1 patient, an EBUS procedure had to be terminated due to severe coughing preventing con- 
tinuation of the procedure. In 1 case, introduction failed due to unspecified reasons.

Four patients complained of pain after an EUS procedure. Two patients developed severe chest pain and $2 \mathrm{had}$ abdominal pain. The cause of pain could not be found in all patients whereupon they were discharged with conservative treatment with pain medication. Six patients complained of a self-limiting sore throat after EUS. Post-procedural fever was seen after 2 examinations. Both received prophylactic antibiotics although no infectious focus was found. Moderate-grade pneumomediastinum with complication-free regression was found in 1 case after a Trucut needle biopsy. Self-limiting nausea with vomiting in 2 patients and intractable coughing, hemoptysis and pain on swallowing in 3 other patients were furthermore reported.

\section{Discussion}

This systematic review shows that the reported complication rate of EUS and EBUS is low with an SAE rate of $0.14 \%$. Fatalities associated with EUS/EBUS have not been described nor have been cases of persistent impairment. Follow-up of patients for possible complications is often not routinely performed, and a lack of documentation may have underestimated the rate of SAE and particularly for minor AE, which are seldom reported. SAE as described in the literature as case reports [5-10] do occur but are rare with an overall rate of $0.14 \%$ and are mostly infectious complications such as mediastinitis and mediastinal abscess formation, which are both severe and potentially life-threatening AE.

Regarding the high number of articles including patients with (suspected) lung cancer and the low prevalence of lung cancer-associated SAE, it seems that EUS and EBUS are safe techniques for an intrathoracic investigation of the mediastinum in these patients. Only 2 cases of severe infectious complications have been described in lung cancer patients yet; one was due to technical problems and the other had necrotic lymph nodes. It has been suggested earlier by Aerts et al. [5] that the presence of necrosis in patients with mediastinal nodal metastases of small cell lung cancer could be a risk factor for developing mediastinal infections due to avascularity of the lesion. We argue that in case of suspected tissue necrosis (e.g. inhomogeneous hypoechoic nodal areas on echographic images), the investigator should avoid puncturing of these specific areas and minimize the number of aspirations.

Complication Rate of EBUS and EUS
The low complication rate found corresponds with the low incidence of complications in the three largest metaanalyses of EUS and EBUS reported in the literature [1214]. In addition, the risk of developing a pneumothorax requiring chest drainage after EBUS is small with only 2 cases reported, therefore obviating the need to routinely perform chest X-rays after EBUS-TBNA, which is also reflected by a study reporting on puncturing lung lesions in 60 patients, in which pneumothorax was not once reported [15].

Regarding infectious SAE, there are probably specific patient groups at risk, e.g. a large part of these AE occurred in patients with cysts and sarcoidosis. The risk of aspirating cystic lesions is apparent considering the relatively low prevalence of mediastinal cysts in general and the documented number of mediastinal infections following cyst aspiration. Cystic lesions are also avascular, and an inoculation of bacteria into the cyst most likely leads to local uninhibited bacterial growth due to the inability of the immune system to reach and fight the pathogens there. In case of a suspected mediastinal cyst based on ultrasound features, needle aspiration of the cyst (e.g. anechoic appearance on echography) should certainly be avoided [10]. Wildi et al. [10] reported tissue sampling in mediastinal cysts in 4 patients, and in 3 of them prophylactic antibiotics were administered. Only in the patient receiving no antibiotics a mediastinal infection developed. In our retrospective case series [9] of patients with sarcoidosis, patients had an increased risk of developing mediastinal infection following nodal puncture, perhaps due to an impaired antimicrobial response within the lymph nodes. Whether a true increased risk exists should be further investigated in a large prospective evaluation. Until then, endoscopists should be aware of this potential complication in patients with sarcoidosis and consider administering prophylactic antibiotic treatment.

There was a difference in the amount of SAE between EUS and EBUS in favor of EBUS, but the reason for this discrepancy is not entirely clear. Because infectious complications are rare with EBUS, it may be that inoculation of gastrointestinal tract bacteria in mediastinal lesions or masses offers an increased infection risk as opposed to the large airways, which are less abundant in commensal flora. On the other hand, the rate of infectious complications in a recent Japanese nationwide survey of EBUS-TBNA was quite comparable to the rate of infectious SAE of EUS-FNA in our review of the literature ( 0.19 vs. $0.17 \%)$ [16].

This review shows that incidental perforation of the gastrointestinal tract $(5 / 6,042 ; 0.08 \%)$ may occur due to 
endoscopic investigation, but the data do not suggest a heightened incidence of such AE compared to the rate of perforations for esophagogastroduodenoscopy (0.03\%) [17]. In the literature, the risk of peri- or postprocedural hemorrhage $(0.01 \%)$ seems lower than that in the survey of Asano et al. [16] (0.68\%), for instance, but this may be attributable to reporting bias; minor bleeding at the puncture site is often observed, but seldom requires intervention.

There are several limitations to this study: some pertain to our study design and the others to the quality of the studies included. We did not screen articles twice, therefore we may have missed or falsely included studies. Secondly, we did not fully register all reasons for possible study exclusion in our systematic review. Although this may have offered a very informative insight, it means we do not know exactly how many studies did not report on complications.

Concerning limitations of the studies included, first of all, most prospective studies had a focus on efficacy of the diagnostic techniques and not on complications. Secondly, information on the occurrence of complications is not always dispensed in the articles we analyzed. For one part, some articles (that were excluded before the final analysis) did not report at all whether AE had occurred. For the other part, in the papers that did report on the occurrence of AE/SAE, a follow-up period was not mentioned or specified in half of the patients. Complications such as bleeding will present immediately during or following endosonography, but others, such as infectious complications, develop over time. The lack of an accurate followup may have excluded these AE, which results in an underestimation of the SAE rate. Also, a considerable part of patients undergoes further investigations, such as mediastinoscopy or thoracotomy, shortly after a negative EUS/EBUS. Inadequate follow-up may have falsely at- tributed $\mathrm{AE}$ to these techniques instead of endosonography.

In general, severe complications and those with fatal outcomes are subject to publication bias and may have remained unpublished. Certainly, the low number of minor complications $(0.22 \%$, nearly as low as 1 in every 500 patients) is not reflecting current practice. Almost no minor $\mathrm{AE}$ have been reported although complaints of hemoptysis, sore throat or intractable coughing are common. Finally, it would be interesting to stratify the complication rate according to specific disease conditions of patients, but unfortunately information on the final diagnoses was often not available.

In conclusion, it seems that endosonography is a safe technique for intrathoracic assessment of the mediastinum. Mortality has not been reported and SAE in patients with lung cancer are very rare. The true incidence of SAE may be higher, possibly due to the lack of communication on complications. For sure, the rate of minor AE is severely underestimated. Better monitoring of (severe) adverse events is advised. This pertains especially to patients with sarcoidosis in whom the risk of developing mediastinal infections may be increased.

\section{Acknowledgments}

We thank Olaf M. Dekkers, MD, PhD (Department of Epidemiology, Leiden University Medical Center, Leiden, the Netherlands) for his advice.

\section{Financial Disclosure and Conflicts of Interest}

This was a nonsponsored contribution. The authors report no conflicts of interests.

\section{References}

1 Silvestri GA, et al: Methods for staging nonsmall cell lung cancer: diagnosis and management of lung cancer, 3rd ed: American College of Chest Physicians evidence-based clinical practice guidelines. Chest 2013;143(suppl 5):e211S-e250S.

$\longrightarrow 2$ Vansteenkiste J, et al: Early and locally advanced non-small-cell lung cancer (NSCLC): ESMO Clinical Practice Guidelines for diagnosis, treatment and follow-up. Ann Oncol 2013;24(suppl 6):vi89-vi98.
3 Tremblay A, et al: A randomized controlled trial of standard vs endobronchial ultrasonography-guided transbronchial needle aspiration in patients with suspected sarcoidosis. Chest 2009;136:340-346.

4 von Bartheld MB, et al: Endosonography versus conventional bronchoscopy for the diagnosis of sarcoidosis: the GRANULOMA randomized clinical trial. JAMA 2013;309:24572464.

5 Aerts JG, et al: EUS-FNA of enlarged necrotic lymph nodes may cause infectious mediastinitis. J Thorac Oncol 2008;3:1191-1193.
6 Gamrekeli A, et al: Infection of a bronchogenic cyst after ultrasonography-guided fine needle aspiration. Ann Thorac Surg 2013;95: 2154-2155.

7 Huang CT, et al: A rare constellation of empyema, lung abscess, and mediastinal abscess as a complication of endobronchial ultrasoundguided transbronchial needle aspiration. Eur J Cardiothorac Surg 2011;40:264-265.

8 Moffatt-Bruce SD, Ross P Jr: Mediastinal abscess after endobronchial ultrasound with transbronchial needle aspiration: a case report. J Cardiothorac Surg 2010;5:33. 
-9 von Bartheld M, van der Heijden E, Annema $\mathrm{J}$ : Mediastinal abscess formation after EUSguided FNA: are patients with sarcoidosis at increased risk? Gastrointest Endosc 2012;75: 1104-1107.

10 Wildi SM, et al: Diagnosis of benign cysts of the mediastinum: the role and risks of EUS and FNA. Gastrointest Endosc 2003;58:362368.

11 US Food and Drug Administration. http:// www.fda.gov/safety/medwatch/howtoreport/ ucm053087.htm.

12 Zhang R, et al: Combined endobronchial and endoscopic ultrasound-guided fine needle aspiration for mediastinal lymph node staging of lung cancer: a meta-analysis. Eur J Cancer 2013;49:1860-1867.

13 Chandra S, et al: Diagnostic accuracy of endobronchial ultrasound-guided transbronchial needle biopsy in mediastinal lymphadenopathy: a systematic review and meta-analysis. Respir Care 2012;57:384-391.

14 Micames CG, et al: Endoscopic ultrasoundguided fine-needle aspiration for non-small cell lung cancer staging: a systematic review and metaanalysis. Chest 2007;131:539-548.

-15 Tournoy KG, et al: EBUS-TBNA for the diagnosis of central parenchymal lung lesions not visible at routine bronchoscopy. Lung Cancer 2009;63:45-49.

16 Asano F, et al: Complications associated with endobronchial ultrasound-guided transbronchial needle aspiration: a nationwide survey by the Japan Society for Respiratory Endoscopy. Respir Res 2013;14:50.

17 Chirica M, et al: Esophageal perforations. J Visc Surg 2010;147:e117-e128.

18 Hirdes MM, et al: Performance of EUS-FNA for mediastinal lymphadenopathy: impact on patient management and costs in low-volume EUS centers. Surg Endosc 2010;24:22602267.
19 Peric R, et al: Transesophageal endoscopic ultrasound-guided fine-needle aspiration for the mediastinal staging of extrathoracic tumors: a new perspective. Ann Oncol 2010;21: 1468-1471.

20 Lee YT, et al: Endoscopic ultrasonographyguided fine-needle aspiration in the management of mediastinal diseases: local experience of a novel investigation. Hong Kong Med J 2010;16:121-125.

21 Varadarajulu S, et al: Comparison of EUSguided 19-gauge Trucut needle biopsy with EUS-guided fine-needle aspiration. Endoscopy $2004 ; 36: 397-401$.

22 Bournet B, et al: Early morbidity of endoscopic ultrasound: 13 years' experience at a referral center. Endoscopy 2006;38:349-354.

23 Iwashita T, et al: The yield of endoscopic ultrasound-guided fine needle aspiration for histological diagnosis in patients suspected of stage I sarcoidosis. Endoscopy 2008;40:400405.

24 von Bartheld MB, et al: Endoscopic ultrasound-guided fine-needle aspiration for the diagnosis of sarcoidosis. Endoscopy 2010;42: 213-217.

25 Aithal GP, et al: EUS-guided tissue sampling: comparison of 'dual sampling' (Trucut biopsy plus FNA) with 'sequential sampling' (Trucut biopsy and then FNA as required). Endoscopy $2007 ; 39: 725-730$.

26 Hernandez A, et al: EUS-FNA as the initial diagnostic modality in centrally located primary lung cancers. J Clin Gastroenterol 2007; 41:657-660.

27 Tournoy KG, et al: Transesophageal endoscopic ultrasound with fine needle aspiration in the preoperative staging of malignant pleural mesothelioma. Clin Cancer Res 2008; 14 : 6259-6263.
28 Annema JT, et al: Implementation of endoscopic ultrasound for lung cancer staging. Gastrointest Endosc 2010;71:64-70, 70 e1.

29 Yasuda I, et al: Mediastinal lymph node staging in potentially resectable non-small cell lung cancer: a prospective comparison of CT and EUS/EUS-FNA. Respiration 2009;78: 423-431.

30 Storch I, et al: Endoscopic ultrasound-guided fine-needle aspiration and Trucut biopsy in thoracic lesions: when tissue is the issue. Surg Endosc 2008;22:86-90.

31 Nayar DS, et al: Comparison of propofol deep sedation versus moderate sedation during endosonography. Dig Dis Sci 2010;55:25372544.

32 Eloubeidi MA, Tamhane A: Prospective assessment of diagnostic utility and complications of endoscopic ultrasound-guided fine needle aspiration. Results from a newly developed academic endoscopic ultrasound program. Dig Dis 2008;26:356-363.

33 Hwangbo B, et al: Transbronchial and transesophageal fine-needle aspiration using an ultrasound bronchoscope in mediastinal staging of potentially operable lung cancer. Chest 2010;138:795-802.

34 Navani N, et al: Utility of endobronchial ultrasound-guided transbronchial needle aspiration in patients with tuberculous intrathoracic lymphadenopathy: a multicentre study. Thorax 2011;66:889-893.

35 Bauwens O, et al: Endobronchial ultrasound and value of PET for prediction of pathological results of mediastinal hot spots in lung cancer patients. Lung Cancer 2008;61:356361.

36 Annema JT, et al: Mediastinoscopy vs endosonography for mediastinal nodal staging of lung cancer: a randomized trial. JAMA 2010; 304:2245-2252.

- 37 Garwood S, et al: Endobronchial ultrasound for the diagnosis of pulmonary sarcoidosis. Chest 2007;132:1298-1304. 